\title{
A NEW STABILIZATION OF ADAPTIVE STEP TRAPEZOID RULE BASED ON FINITE DIFFERENCE INTERRUPTS*
}

\author{
J. ALEX LEE ${ }^{\dagger}, J_{A E W O O K ~ N A M} \ddagger$, AND MATTEO PASQUALI ${ }^{\S}$
}

\begin{abstract}
The adaptive step trapezoid rule (TR) is a generally effective numerical integrator, but it is prone to ringing instability and solution stall. We introduce a new stabilization algorithm based on finite difference interrupts (FDI) that reduces ringing and prevents stall. Unlike previously reported stabilization schemes, our algorithm achieves stability and second order accuracy without incurring significant computational cost or spurious diffusion. TR with FDI is at least as stable and accurate as existing methods when solving the prototypical scalar problem. We demonstrate that it has better stability, accuracy, and cost when solving the tightly coupled system describing free surface flows of viscoelastic liquids. Though we demonstrate TR with FDI in the context of a finite element method-of-lines, it is applicable to any TR-based algorithm.
\end{abstract}

Key words. trapezoid rule, Adams-Bashforth-Moulton, method-of-lines, adaptive time step, fluid mechanics, finite element method, ringing instability, stiff PDE

AMS subject classifications. $35,65,76$

DOI. $10.1137 / 140966915$

1. Background and context. Transient free surface flows of incompressible complex fluids arise in numerous fluid-phase manufacturing processes such as film coating, printing, and fiber spinning. The solution of the relevant coupled set of partial differential equations remains challenging for various reasons. Here, we focus on the numerical stability of a method-of-lines approach using the finite element method (FEM) and the trapezoid rule (TR) with adaptive time stepping [33, 7]. The FEM has emerged as the method of choice for space discretization of capillarity-dominated free surface flow problems [47, 57, 13], especially for viscoelastic flows [7, 1, 48, 8, 5, 39].

Many sophisticated time integrators have been developed for solving stiff coupled problems in structural and fluid mechanics $[15,30,55,56,17,26,54,53,22]$, but we restrict our discussion to the relatively simple TR and other linear multistep methods (LMM). TR is often advocated for its $A$-stability and optimal accuracy [21], as well as its amenability to error-controlled adaptive time stepping [33, 49, 32]. However, it is also known to suffer "ringing" instability, which often stalls the integration.

*Submitted to the journal's Methods and Algorithms for Scientific Computing section April 28, 2014; accepted for publication (in revised form) December 22, 2014; published electronically March 19, 2015. This work was supported by NSF NSEC grants CMMI-0531171 and CMMI-1031171, and the computations were supported by the Data Analysis and Visualization Cyberinfrastructure funded by NSF grant OCI-0959097; by NIH award NCRR S10RR02950 and an IBM Shared University Research (SUR) Award in partnership with CISCO, Qlogic, and Adaptive Computing; and by the Shared University Grid at Rice funded by NSF grant EIA-0216467 and a partnership between Rice University, Sun Microsystems, and Sigma Solutions, Inc.

http://www.siam.org/journals/sisc/37-2/96691.html

$\dagger$ Department of Chemical and Biomolecular Engineering, Rice University, Houston, TX 77005 (jalexlee@rice.edu).

${ }^{\ddagger}$ School of Chemical Engineering, Sungkyunkwan University, Suwon, Korea (jaewooknam@skku.edu). This author's research was supported by National Research Foundation of Korea grant NRF-2013R1A1A1004986.

$\S$ Corresponding author. Department of Chemical and Biomolecular Engineering, Department of Chemistry, Ken Kennedy Institute for Information Technology, and the Smalley Institute for Nanoscale Science and Technology, Rice University, Houston, TX 77005 (mp@rice.edu). 
Ironically, large step sizes deemed appropriate by the adaptive step algorithm are one of the triggers for activating the ringing instability.

The nature of this ringing and strategies for mitigating its effects have been discussed extensively in the literature $[40,46,45,58,27,20,10]$, especially in the context of the second order finite difference method-of-lines (the well-known CrankNicolson method [19]) [11, 41, 42], and more recently of the finite element method-oflines $[33,2,50,37,24,25,52]$. For our purpose, the most relevant method is the time step averaging (TSA) method due to Gresho, Griffiths, and Silvester [31], a simple and inexpensive scheme that effectively stabilizes the solution, albeit with compromised accuracy. It has been demonstrated with finite element space discretization on fixed-domain fluid flow problems $[37,50]$ and further extended to nonisothermal flows $[24,52]$; however, we show that TSA loses efficacy when solving viscoelastic free surface flow problems.

We introduce the finite difference interrupt (FDI) and show that it successfully stabilizes viscoelastic free surface flow computations while preserving second order accuracy. The remainder of this section briefly reviews TR, its ringing instability, and other relevant stable LMMs. In section 2, we define the TR-FDI method and discuss its properties and performance on the scalar ODE test problem. In sections 3 and 4, we apply TR-FDI on the transient lid-driven cavity flow and the viscoelastic filament stretching flow. Our results demonstrate that the relatively inexpensive TRFDI is a robust, efficient, and accurate TR stabilization scheme.

Review of TR. The well-known TR with adaptive time stepping arises from the second order Adams-Bashforth-Moulton (ABM) predictor-corrector pair of LMMs $[3,33]$. Its implementation is independent of the governing equation or the spatial discretization techniques, and the key points are reviewed below for the general scalar ODE $f(y, \dot{y})=0$. Given the solution $y_{k}$ and its time derivative $\dot{y}_{k}$ at any time step $t_{k}$, TR gives for the next time step (defining $\Delta t_{k+1}=t_{k+1}-t_{k}$ )

$$
y_{k+1}=y_{k}+\frac{\Delta t_{k+1}}{2}\left(\dot{y}_{k}+\dot{y}_{k+1}\right)
$$

which can be rearranged to isolate the TR derivative

$$
\dot{y}_{k+1}=\frac{2}{\Delta t_{k+1}}\left(y_{k+1}-y_{k}\right)-\dot{y}_{k} .
$$

In TR, (1.2) replaces $\dot{y}$ in the ODE to yield an algebraic equation for the unknown $y_{k+1}$ in terms of the known $y_{k}$ and $\dot{y}_{k}$. For nonlinear problems, an initial guess for the implicit TR solution (corrector) is provided by the explicit second order AdamsBashforth (AB2) solution (predictor)

$$
y_{k+1}^{\mathrm{P}}=y_{k}+\frac{\Delta t_{k+1}}{2}\left[\left(2+\frac{\Delta t_{k+1}}{\Delta t_{k}}\right) \dot{y}_{k}-\frac{\Delta t_{k+1}}{\Delta t_{k}} \dot{y}_{k-1}\right] .
$$

Thus, only the solutions at two time steps need to be stored in memory when implementing TR. Following a nonlinear solve, the TR derivative $\dot{y}$ is computed from (1.2); because it is recursively defined, this quantity must also be propagated in time, requiring storage for three time steps. Adaptive time stepping is achieved by controlling the local truncation error (LTE) associated with each time step, determined by comparing the TR and AB2 solutions. Taylor series analysis yields the LTE estimator [32, 49]

$$
\left\|e_{k+1}\right\|=\frac{\left\|\delta_{k+1}\right\|}{3\left(1+\frac{\Delta t_{k}}{\Delta t_{k+1}}\right)},
$$


where $\left\|\delta_{k+1}\right\|$ is the magnitude of the difference between the AB2 and TR solutions. For the systems of ODEs arising from the method-of-lines discretization of a PDE, $\|\cdot\|$ is an appropriately defined vector norm $[49,33,31]$. The choice of this norm will affect the value of the LTE tolerance required to achieve some global accuracy, but it is not expected to change the qualitative behavior of the adaptive time stepping algorithm. At successive time steps, the ratio $\left\|e_{k+2}\right\| /\left\|e_{k+1}\right\|$ is proportional to $\left(\Delta t_{k+2} / \Delta t_{k+1}\right)^{3}$, implying that the LTE of the next step can be controlled by setting a user-specified tolerance $\left\|e_{k+2}\right\| \leq \varepsilon$ and selecting the next time step size [32, 49]

$$
\Delta t_{k+2}=\Delta t_{k+1}\left(\frac{\varepsilon}{\left\|e_{k+1}\right\|}\right)^{1 / 3} .
$$

Only the dynamic variables are used in the LTE estimator when solving a coupled system of equations. For example, when solving the Navier-Stokes equations of incompressible flow, the pressure variable is ignored because it is algebraic in time [33].

Ringing instability. TR is susceptibile to "ringing," or spurious oscillations in time. When applied to the prototypical scalar problem $\dot{y}=\gamma y$, TR with constant time step size yields an explicit form of the solution $y_{k}=\xi^{k} y_{0}$, with growth factor $\xi$

$$
\xi=\frac{1+\gamma \Delta t / 2}{1-\gamma \Delta t / 2}
$$

TR is $A$-stable as defined by the property that $|\xi|<1$ for $\operatorname{Re}(\gamma \Delta t)<0$ [21]. It is not $L$-stable, a property that further requires $\xi \rightarrow 0$ in the limit of large step size $\operatorname{Re}(\gamma \Delta t) \rightarrow-\infty$. In this limit, (1.6) gives $\xi \rightarrow-1$ so that TR solutions grow asymptotically like $(-1)^{k}$ to produce step-by-step ringing. Oscillations are sensitive to the initial conditions of the numerical system and can be controlled to some extent by taking several backward Euler (BE) steps initially [2, 11, 41].

Solution ringing results when time step sizes are too large, but it can in turn induce stall for an adaptive time stepping algorithm: time step size fails to grow even when the physics allow it, often at time step sizes orders of magnitude smaller than necessary. Ringing-induced stall is typical in an unsteady system approaching steady state and often occurs following abrupt temporal changes (as by forcing terms). The ringing instability may worsen for stiffer problems $[37,42,52]$ as well as problems where space-time coupling is stronger.

Stable and stabilized LMMs. The backward difference formulae (BDF) constitute the other class of implicit LMMs relevant to our purpose. In the second order BDF2 (also known as Gear's method [29]), the three-point backward finite difference at $t_{k+1}$

$$
\dot{y}_{k+1}^{\mathrm{bd}}=\frac{\alpha^{2} y_{k-1}-(1+\alpha)^{2} y_{k}+(1+2 \alpha) y_{k+1}}{\Delta t_{k+1}(1+\alpha)}, \quad \alpha=\frac{\Delta t_{k+1}}{\Delta t_{k}},
$$

replaces $\dot{y}$ in the ODE to yield an algebraic equation for the unknown $y_{k+1}$ in terms of the previously solved $y_{k}$ and $y_{k-1}$. Like TR, BDF2 is amenable to LTE-controlled adaptive time stepping [33]. Because the BDF is not recursively defined in (1.7), the quantity $\dot{y}$ does not need to be stored or propagated forward in time; however, the adaptive step BDF2 algorithm does require storage of the solution at one more prior time step, namely, $y_{k-2}$, for calculation of the predictor. BE is the first order method in both the ABM and BDF families and is written 


$$
\dot{y}_{k+1}^{\mathrm{BE}}=\frac{y_{k+1}-y_{k}}{\Delta t_{k+1}} .
$$

$\mathrm{BE}$ and BDF2 are both $L$-stable and suffer neither the ringing instability nor adaptive time step stall. BE is generally unsuitable for high performance codes because of its low accuracy and highly diffusive nature, i.e., it damps solutions even when the ODE is unstable. BDF2 is a reasonable alternative to TR with its second order accuracy and comparable computational cost but is less preferable for convection-dominated problems because it is diffusive like BE, though not as aggressively [33].

In the hybrid TR-BDF2 [4], each time step is split into two substeps taken by TR and BDF2 in succession. This well-studied method is also $L$-stable, and the optimal substep division yielding the lowest LTE (and the only case considered here) is $(2-\sqrt{2}) \Delta t[4,35,23]$. Several different LTE estimators for use in adaptive time stepping are discussed in [4]. TR-BDF2 is attractive because it is less diffusive and more accurate than BDF2. However, it requires significant modification to an existing TR-based code and requires a costly additional nonlinear solve per time step.

The preceding analysis of TR based on (1.6) suggests that it can be stabilized by removing contributions to the solution of the form $(-1)^{k}$ [31]. The TR-TSA scheme $[31,37,24,52,50]$ accomplishes this by intermittently averaging the most recent trio of solutions and derivatives to define a new pair at time steps half way between the old ones; this is essentially a linear interpolation (see Appendix A). Unlike TR-BDF2, TR-TSA adds virtually no computational cost to the underlying TR algorithm and has been shown to effectively stabilize adaptive time step stall in fluid flow problems [37]. The drawback is that overall solution accuracy is expected to be degraded because of TSA's first order accuracy; however, detailed accuracy assessments were not reported.

2. TR with finite difference interrupts. Our stabilization is based on the same premise as that of the TR-TSA scheme [31], which is to eliminate the asymptotic $\xi \rightarrow-1$ behavior. We propose to remove the recursion of the TR derivative $\dot{y}$ on its previous value in (1.2) by modifying $\dot{y}_{k+1}$ after it is solved, such that for the next step

$$
\dot{y}_{k+2}=\frac{2}{\Delta t_{k+2}}\left(y_{k+2}-y_{k+1}\right)-\dot{y}_{k+1}^{\bmod } .
$$

The FDI replaces the last computed TR derivative with the backward finite difference formula (1.7), i.e., setting $\dot{y}_{k+1}^{\text {mod }}=\dot{y}_{k+1}^{\text {bd }}$ in (2.1). Although storage of the solution vector is not typically limiting, we note that FDI requires the solution at one extra time step $\left(y_{k-1}\right)$ to be kept in memory.

TR-FDI is closely related to BDF2 and the hybrid TR-BDF2 but is distinct from both: (1.7) is evaluated retrospectively from the solution $y_{k+1}$ of the implicit equations arising from TR, rather than being substituted into the governing equation to yield the implicit BDF2 equation for $y_{k+1}$. It is also related to TR-TSA, which can be interpreted as a first order variant of FDI. Both are trivial to implement in an existing TR-based code and add negligible computational cost. In contrast, TRBDF2 requires a full BDF2 solver that incurs a costly extra nonlinear solve per time step. TR-FDI is simpler than TR-TSA in that FDI does not shift time steps, and it is expected to be more accurate because it is based on a higher order finite difference.

Stability analysis. The notation TR-TSA- $n$ and TR-FDI- $n$ means that TSA or FDI is applied at fixed intervals of $n$ time steps. To achieve a good compromise between accuracy and stability, TR-TSA should be implemented every 5 to 10 steps $[37,24]$, yielding TR-TSA-05 through TR-TSA-10. When TR-FDI-01 is applied 

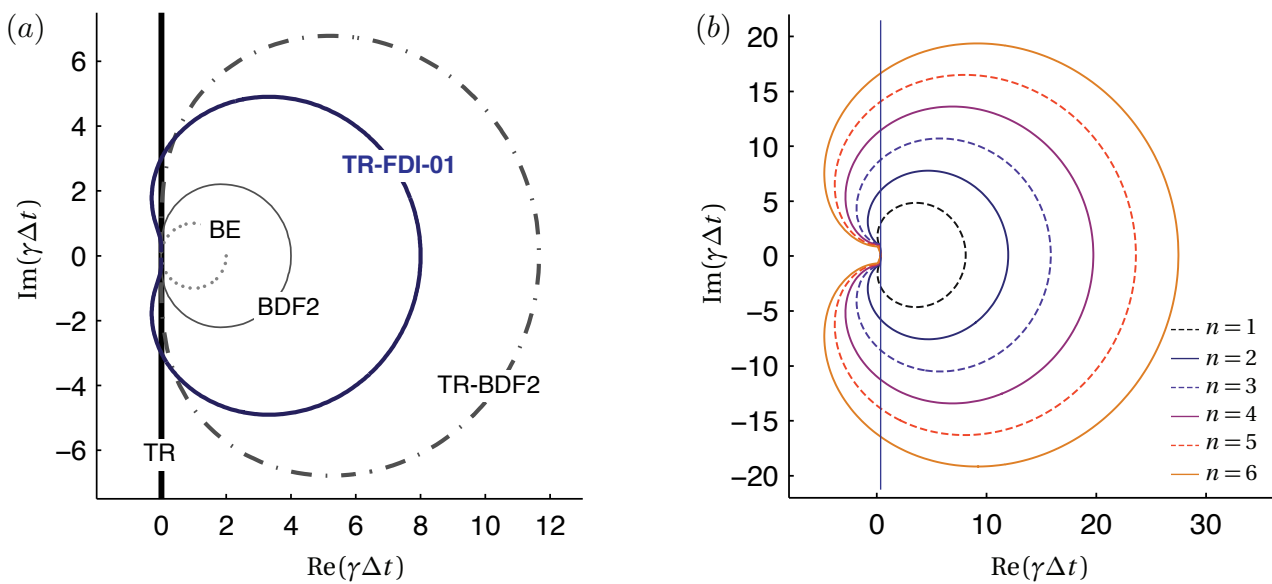

FIG. 1. Stability regions enclosing $\max |\xi|>1$. (a) Stability plots for TR, BE, BDF2, TR-BDF2, and TR-FDI-01. (b) Stability plots for TR-FDI- $n$ with $1 \leq n \leq 6$.

to the prototypical scalar ODE $\dot{y}=\gamma y$ with constant step size $\Delta t$, the growth factor $\xi$ in the explicit form of the solution $y_{k}=\xi^{k} y_{0}$ satisfies the third order polynomial characteristic equation (Appendix B)

$$
0=-\xi^{3}-\frac{7 \xi^{2}}{2 \gamma \Delta t-4}+\frac{2 \xi}{\gamma \Delta t-2}-\frac{1}{2 \gamma \Delta t-4},
$$

which has three roots. Similarly, BDF2 has two roots, and BE, TR, and TR-BDF2 have one. Methods without spurious roots are sometimes praised, but the multiplicity of roots is not an important issue in practice [36]. Due to time step shifting, the characteristic equations for TR-TSA- $n$ cannot be derived for comparison.

All three roots of TR-FDI-01 satisfy $|\xi| \rightarrow 0$ as $\gamma \Delta t \rightarrow-\infty$, indicating that TRFDI-01 is stable with respect to ringing. However, its stability plot in Figure 1(a) shows lobes of instability $(\max |\xi| \geq 1)$ extending into the left half plane, demonstrating that it is not $A$-stable and therefore by definition not $L$-stable; rather, it appears to be stiffly stable $[28,18]$. In other words, TR-FDI-01 does not asymptotically introduce spurious oscillations into the solution but may spuriously amplify existing ones in a certain range of frequencies or time step sizes.

In general, the characteristic equations for TR-FDI- $n$ are polynomials of order $n+2$ (see Appendix B). All roots are shown to vanish asymptotically for these polynomials up to $n=6$, suggesting that TR-FDI- $n$ is generally at least stiffly stable and resistant to ringing. The stability plots in Figure 1(b) show that TR-FDI- $n$ is generally $A$-unstable with larger lobes of instability as $n$ increases. The following section demonstrates that $A$-instability is practically an unimportant consideration, as suggested originally in [21].

Practical implications of $\boldsymbol{A}$-instability. We explore the spurious amplification of frequencies within the lobes of $A$-instability (Figure 1(b)) by solving the neutrally stable oscillatory scalar problem $\dot{y}=i \omega y$. Fitting the expected form of the true solution $\operatorname{Re}(y)=\exp (a t) \cos (b t)$ to the numerical one provides as a measure of $A$-stability the spurious growth constant $a$. This measure is plotted for each method in Figure 2(a) as a function of step size. 
(a)

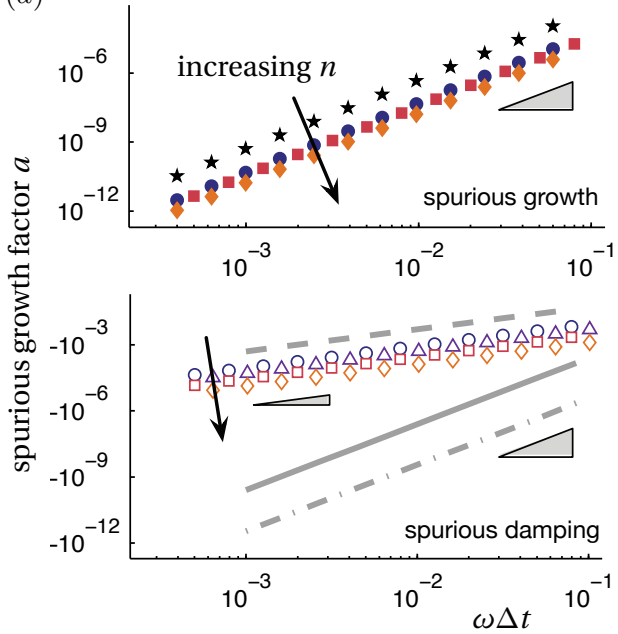

(b)

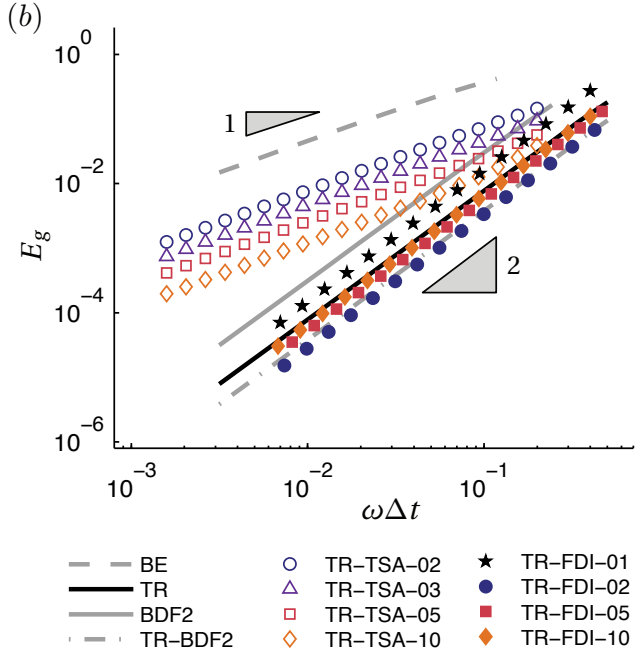

FIG. 2. (a) Spurious growth constant for various integration methods. (b) Global errors $E_{g}=$ $\left|\operatorname{Re}\left(y_{k}\right)-\cos \left(t_{k}\right)\right|_{\infty}$ as a function of dimensionless step size $\omega \Delta t$.

TR is neutrally $A$-stable $(a \approx 0)$, and BE, BDF2, and TR-BDF2 are all spuriously damping $(a<0)$. BE is the most aggressively damping method with the largest $|a|$. As expected from the stability analysis, BDF2 is less damping than BE and TR-BDF2 is least damping. TR-TSA- $n$ can be more damping than BDF2, but not as much as $\mathrm{BE}$; its damping magnitude scales with $\omega \Delta t$ similarly to that of $\mathrm{BE}$.

TR-FDI- $n$ spuriously amplifies the oscillations $(a>0)$, demonstrating that it is $A$-unstable; the method becomes more neutral as $n$ increases. The magnitude and scaling of spurious amplification by TR-FDI- $n$ are similar to those of damping by BDF2 or TR-BDF2 (Figure 2(a)). Thus, from a practical perspective, TR-FDI- $n$ appears to be as good as the more expensive TR-BDF2 and better than the more strongly damping BDF2 and TR-TSA- $n$.

The maximum global error $E_{g}=\left|\operatorname{Re}\left(y_{k}\right)-\cos \left(t_{k}\right)\right|_{\infty}$ scales with the dimensionless time step size like $E_{g} \sim(\omega \Delta t)^{q}$, where $q$ is the method's order of accuracy. Figure 2(b) shows the global errors computed for the various integration methods, verifying first order accuracy of BE and second order accuracies of TR, BDF2, and TR-BDF2. The hybrid TR-BDF2 has lower global errors than TR, indicating that it better preserves the principal oscillation frequency despite spuriously damping it.

The global errors for TR-TSA- $n$ lie between those of BE and TR, decreasing as $n$ is increased (TSA is applied less frequently). The linear scaling with time step size $(q=1)$ indicates that TR-TSA- $n$ is overall first order accurate. However, the second order accuracy of the underlying TR must be recovered in the limit $n \rightarrow \infty$, or practically when $n$ is greater than the number of time steps required to reach termination. When TSA is applied infrequently enough, for example, $n=10$ with $\omega \Delta t \approx 5 \times 10^{-2}$, the order of accuracy is intermediate (Figure 2(b)).

Unlike TR-TSA- $n$, TR-FDI- $n$ preserves second order accuracy for all $n$ and $\omega \Delta t$ considered. Except for the special case of $n=1$, TR-FDI- $n$ has lower global error values than the underlying TR, with accuracy diminishing toward that of TR as $n$ increases; this is in contrast to the accuracy of TR-TSA- $n$, which improves monotonically as $n$ increases. TR-FDI-02 has similar accuracy to TR-BDF2, perhaps reflecting 

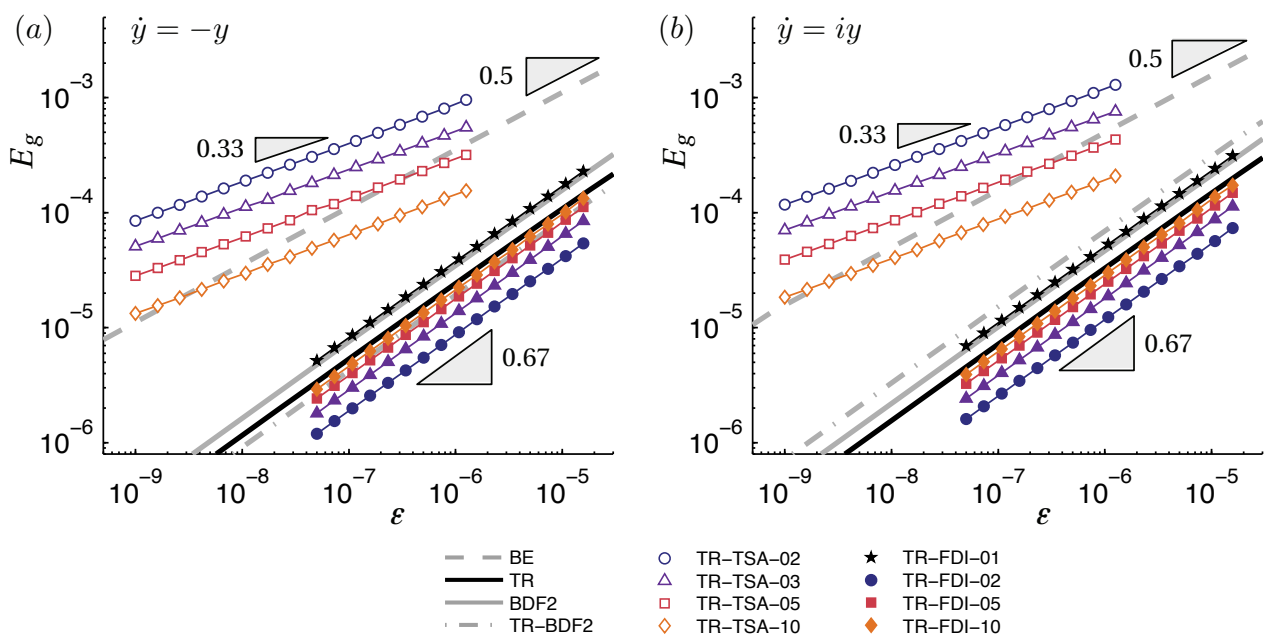

FIG. 3. Maximum error $E_{g}$ of the numerical solutions to the test problems (a) $\dot{y}=-y$ and (b) $\dot{y}=i y$. The power law exponent for a qth order accurate method is $q /(q+1)$.

the procedural similarity of alternating TR and BDF2 derivatives. TR-FDI-01 resembles BDF2 in both procedure and accuracy.

Performance of TR-FDI with adaptive step. The integration methods are compared in the adaptive time stepping context on the ODE test problem $\dot{y}=\gamma y$ with $\gamma=-1$. The maximum global errors $E_{g}=\left|y_{k}-\exp \left(\gamma t_{k}\right)\right|_{\infty}$ are plotted in Figure 3(a) with respect to the LTE tolerances $\varepsilon$, which dictate the variable time step sizes by controlling the accumulation of LTEs. For algorithms with order of accuracy $q$, global error scales as

$$
E_{g}=c_{E} \varepsilon^{\frac{q}{q+1}}
$$

with proportionality constant $c_{E}$. Thus, for a first order accurate method $E_{g} \sim \varepsilon^{1 / 2}$ and for a second order method $E_{g} \sim \varepsilon^{2 / 3}$ [33]. BE, BDF2, TR, and TR-BDF2 all exhibit the expected orders of accuracy according to Figure 3(a).

TR-TSA- $n$ is expected to be at least first order accurate, but Figure 3(a) shows that $E_{g} \sim \varepsilon^{0.33}$, corresponding to an order of accuracy of only $q \approx 0.5$. TR-TSA- $n$ is thus less accurate than BE below a critical $\varepsilon$ or $n$. Conversely, the underlying second order accuracy of TR must again be recovered for large enough $n$ and $\varepsilon$; for this reason, [31] states that large $n$ must be used with higher (looser) tolerances. The reduced order of accuracy is explained by the fact that TSA is essentially an oversized BE step with an oversized LTE. The subsequent step size is also corrupted because the LTE estimator (1.4) is incorrect following a TSA.

The LTE is similarly corrupted following an FDI, but TR-FDI- $n$ remains second order accurate. This is at least in part due to the second order accuracy of the BDF2 derivative estimate and the fact that FDI never alters the solution by time step shifting. Generalizing to complex $\gamma=-\gamma_{0}+i \omega$ (including $\gamma_{0}=0$ ) gives quantitatively similar results for all methods $(\gamma=i$ shown in Figure 3(b)); TR-FDI- $n$ is always second order accurate despite its $A$-instability.

The number of time steps $N_{t}$ necessary to achieve the termination criterion measures a method's computational economy and is shown in Figure 4(a) as a function 

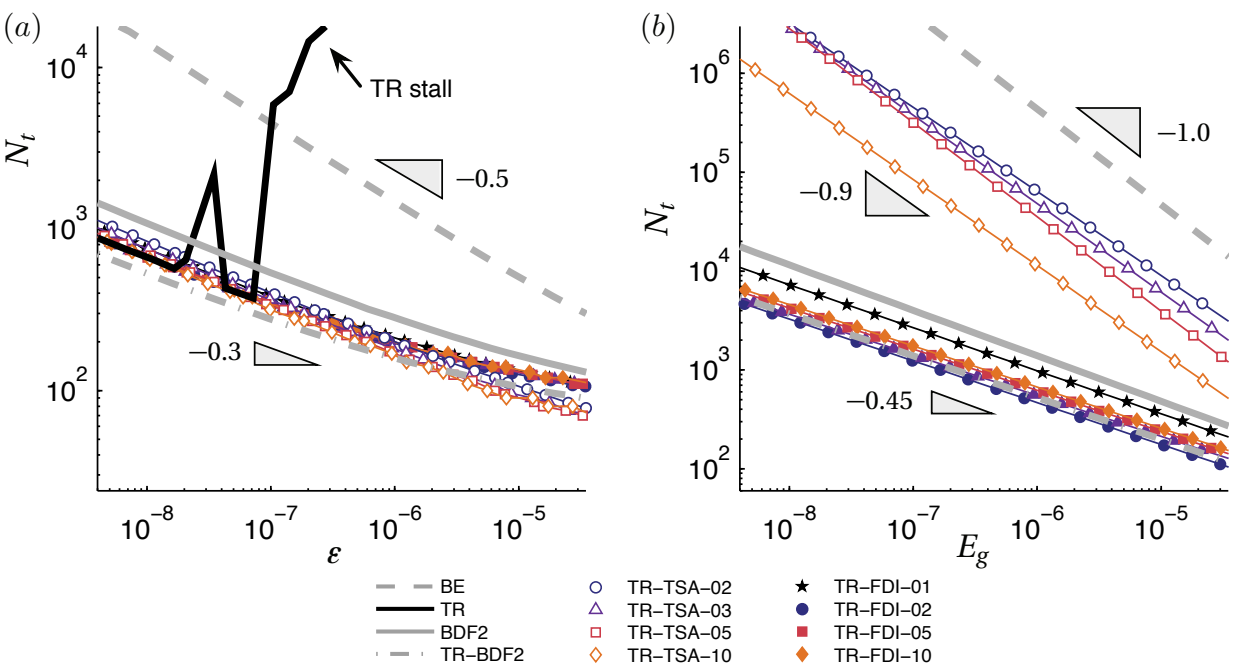

FIG. 4. Number of time steps $N_{t}$ required to achieve steady state $\left(\dot{y}<10^{-11}\right)$ for the test problem $\dot{y}=-y$ as a function of (a) the LTE tolerance $\varepsilon$ and (b) maximum global error $E_{g}$.

of the LTE tolerance $\varepsilon$. Instability-induced stall prevents TR from reliably achieving steady state for large $\varepsilon$, but the instability is relieved as the tolerance is tightened [33]. BE is least economical except when compared to the unstable TR. TR-BDF2 appears most economical but requires an extra nonlinear solve per time step. The stabilized TR-TSA- $n$ and TR-FDI- $n$ perform similarly, yielding $N_{t}$ within a factor of two. Another practical performance measure is the efficiency, i.e., how $N_{t}$ scales with the LTE tolerance, and can be characterized using the power law

$$
N_{t}=c_{N} \varepsilon^{-\chi}
$$

with proportionality constant $c_{N}$ and efficiency coefficient $\chi$ (lower is better). The nominally second order methods are more efficient $(\chi=0.3)$ than $\mathrm{BE}(\chi=0.5)$.

Figure 4(a) shows the economy and efficiency of each method on the basis of LTE tolerance, but such a comparison is misleading because of the large disparity in the global accuracies (Figure 3). Moreover, LTE-based step size control is increasingly questionable as TSA or FDI is applied more frequently and the LTE estimator (1.4) is rendered less appropriate. We propose a fairer comparison in Figure 3(b) made at fixed "global error tolerance." Although global error cannot actually be controlled (only a tolerance on local error is imposed), the power law fits to (2.3) and (2.4) from the results in Figures 3(a) and 4(a) can be combined to estimate the value of $N_{t}$ satisfying an approximate global error tolerance,

$$
N_{t}=\left(c_{N} c_{E}^{\bar{\chi}}\right) E_{g}^{-\bar{\chi}},
$$

with adjusted efficiency coefficient $\bar{\chi}=\chi(q+1) / q$. Figure $4(\mathrm{~b})$ illustrates that when comparing the methods on the basis of global errors, TR-FDI- $n$ is actually more efficient than TR-TSA- $n$ due to its second order accuracy. It is comparable to BDF2 and TR-BDF2 but requires minimal modifications to a TR-based code and adds virtually no additional computational cost.

In brief, FDI is a simple and inexpensive modification to TR that stabilizes against adaptive time step stall while preserving second order accuracy. Applied to the scalar 


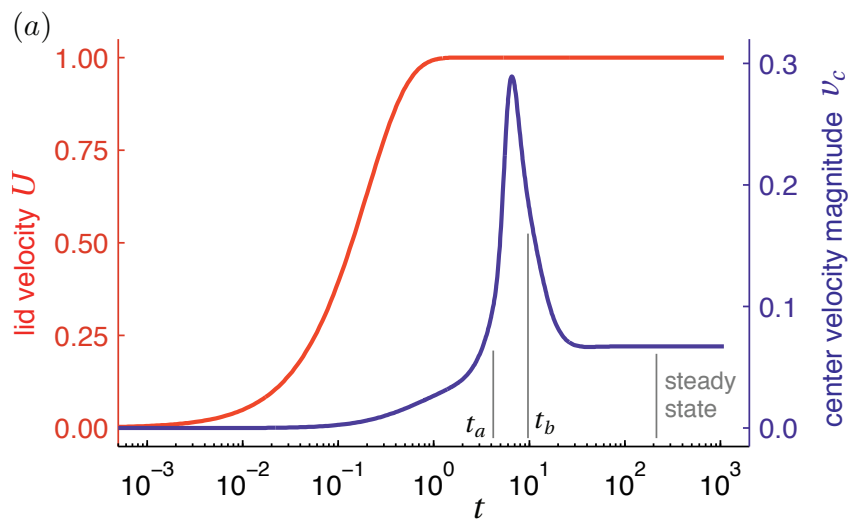

(b)

FIG. 5. (a) Evolutions of driving lid velocity (left axis) and center velocity magnitude (right axis). (b) Steady state streamlines and magnitude of the velocity field.

ODE test problem, the stiffly stable TR-FDI performs comparably to or better than existing methods of similar or greater complexity. In the following, we compare its efficacy on PDEs in the FEM method-of-lines context.

3. Transient lid-driven cavity. TR-FDI- $n$ is applied to the two-dimensional (2D) transient lid-driven cavity flow [51,37, 38], a classical benchmark fluid mechanics problem in which the fluid is set into a recirculating flow inside a cavity by a sliding lid. The velocity $\mathbf{v}$ and pressure $p$ are governed by the incompressible Navier-Stokes equations of momentum and mass conservation,

$$
\begin{aligned}
\rho\left(\frac{\partial \mathbf{v}}{\partial t}+\mathbf{v} \cdot \nabla \mathbf{v}\right) & =\nabla \cdot \mathbf{T}+\rho \mathbf{g}, \\
0 & =\nabla \cdot \mathbf{v},
\end{aligned}
$$

with Newton's constitutive law for the fluid state of stress,

$$
\mathbf{T}=-p \mathbf{I}+\eta_{s}\left[\nabla \mathbf{v}+(\nabla \mathbf{v})^{T}\right],
$$

and no-slip velocity boundary conditions along the cavity walls and sliding lid, which has transient velocity $U(t)$. The only material parameters are the fluid density $\rho$ and viscosity $\eta_{s}$. Gravity is neglected $(\mathbf{g}=\mathbf{0})$. The velocity and pressure are discretized in space by a standard Galerkin FEM with quadrilateral $Q_{2} P_{-1}$ [33] elements. The cavity domain is discretized into a $96 \times 96$ mesh with square elements $(102,146$ total degrees of freedom) decreasing in size toward the cavity corners to better capture the secondary vortices there.

We adopt the parameters in [37] for direct comparison: square cavity with side length $\ell$, steady state lid velocity $U_{\infty}$, and parameters $\rho$ and $\eta_{s}$ chosen to set the steady state Reynolds number $\operatorname{Re}_{\infty}=\rho U_{\infty} / \eta_{s}=1000$, where Re $\gg 1$ indicates strong convection and higher susceptibility to ringing. The transient lid velocity is $U(t) / U_{\infty}=1-e^{-5 t}$, where time $t$ has been scaled by $\ell / U_{\infty}$. The lid and center velocity evolutions are illustrated in Figure 5(a). The initial time step size is set to $\Delta t_{0}=10^{-16}$. We note that [37] used triangular $P_{2} P_{1}$ Taylor-Hood elements with similar resolution $(64 \sim 128$ element edges per cavity side). 

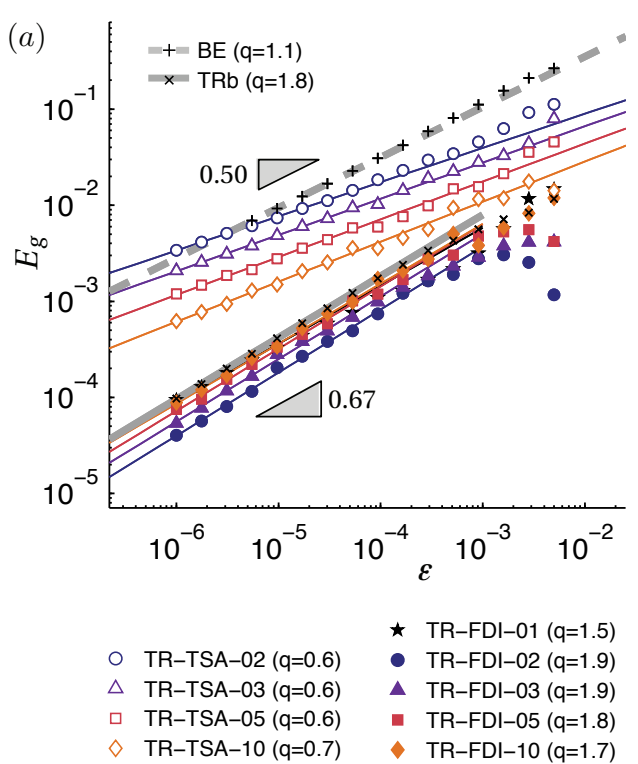

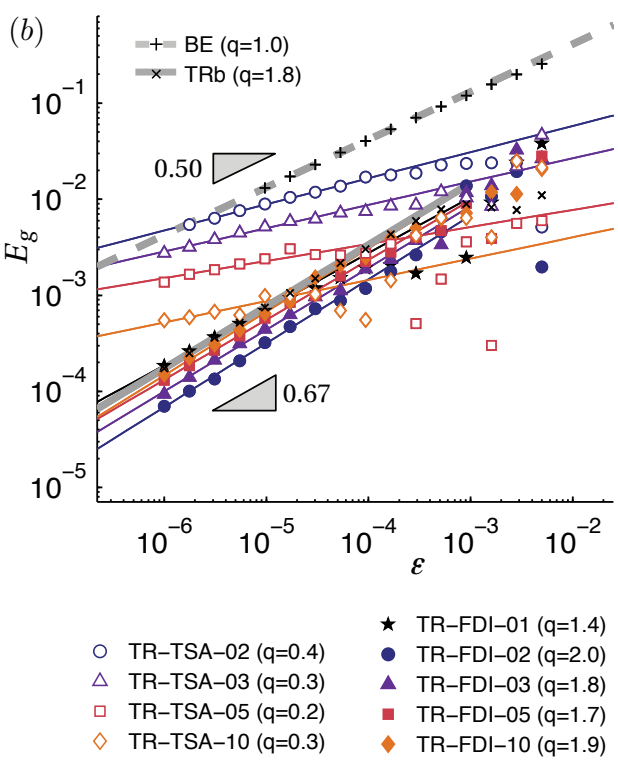

FIG. 6. Accumulated time discretization error $E_{g}=\left(v_{c}-v_{c, 0}\right) / v_{c, 0}$ with respect to LTE tolerance $\varepsilon$ at times (a) $t_{a}=4$ and (b) $t_{b}=9.5$. Lines are fits to (2.3) with $q$ given in the legend.

The solution is characterized by the velocity magnitude at the cavity center $v_{c}(t)$, which shows a clear transient from $0.1<t<30$ before approaching steady state around $t>200$ (Figure 5(a)). Because this end time is not known a priori, the algorithm checks for a steady state criterion for termination, defined here as when the norm of the velocity derivative falls below a threshold.

Results. Hereafter we do not consider BDF2 or TR-BDF2, which require significant alteration of our TR-based fluid flow solver. TR-FDI- $n$ is compared to BE, TR, and TR-TSA- $n$. Error analysis is carried out at two times, ${ }^{1} t_{a}=4.0$ and $t_{b}=9.5$ where the solution metric $v_{c}$ undergoes rapid change (Figure $5(\mathrm{a})$ ), i.e., where time step control is most important. TR with initial time step size increased to $\Delta t_{0}=10^{-9}$ (denoted TRb) is less susceptible to stall, reaching at least $t \geq t_{b}$ within the maximum number of time steps; neither achieves steady state due to instability induced stall.

Numerical solutions of PDEs are subject to spatial discretization errors as well as time discretization errors. However, insofar as differently meshed problems in the method-of-lines are simply different sets of ODEs (space and time discretization are decoupled), the notion of an ostensible "true" solution in the limit $\Delta t \rightarrow 0$ (or $\varepsilon \rightarrow 0$ ) for a fixed spatial discretization is applicable in the ODE sense. Such an extrapolated solution of the center velocity magnitude is used to define the total accumulated time discretization error $E_{g}=\left(v_{c}-v_{c, 0}\right) / v_{c, 0}$, which is expected to follow (2.3) as before. Figure 6 plots $E_{g}$ with respect to $\varepsilon$ at times $t_{a}$ and $t_{b}$ and verifies that BE is first order accurate and that TRb is second order accurate $(q \approx 1.7)$. TR-TSA- $n$ is less than first order accurate $(0.3<q<0.6)$, while TR-FDI- $n$ remains second order accurate $(1.3<q<1.8)$.

\footnotetext{
${ }^{1}$ Because the time step size selection is adaptive, it is not possible to specify exactly the solution times; solutions at a specific time $t$ are obtained by an interpolation method consistent with TR [33].
} 
(a)

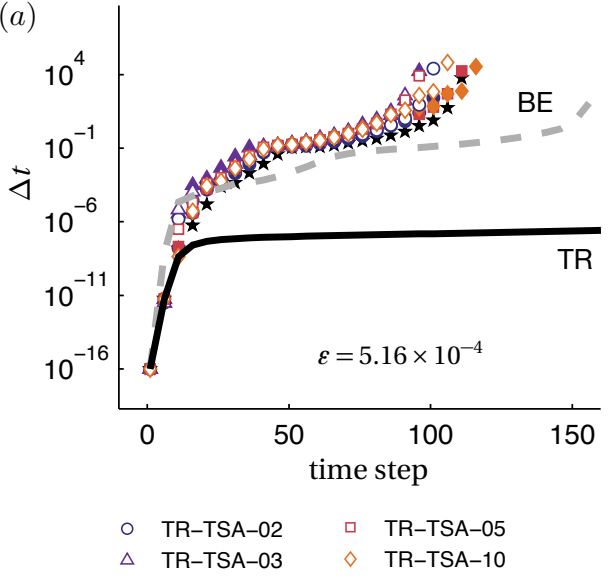

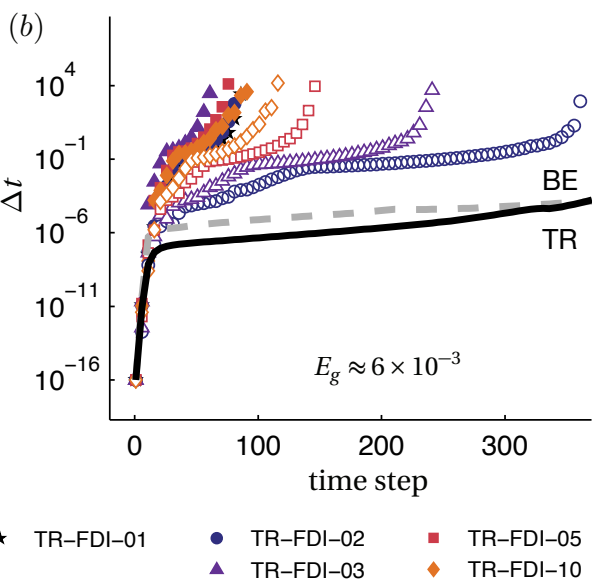

FIG. 7. Time step size progressions with respect to fixed (a) LTE tolerance $\varepsilon=5.16 \times 10^{-4}$ and (b) accumulated time discretization error $E_{g} \approx 6 \times 10^{-3}$ (as determined at $t=t_{a}$ ).

Figure 7(a) shows time step size growth at fixed LTE tolerance $\varepsilon$ and can be compared to the results in [37]. Pure TR stalls at early time steps, while all other methods take progressively larger steps as allowed by the physics. The time step size growth by BE is less rapid than by TR-TSA- $n$ and TR-FDI- $n$, which appear to take an optimal number of steps when $3<n<10$; for TR-TSA- $n$, this agrees with the results and recommendations of [37]. Figure 7(b) compares time step size growth at fixed $E_{g}$ (approximately) to more clearly illustrate the relative efficiencies.

To ensure fair comparison, the number of time steps $N_{t}$ to reach steady state is measured by the criterion $t \geq 200$ rather than a criterion on the velocity derivatives, which are different for each method (TR, TSA, FDI). Figure 8(a) shows $N_{t}$ as a function of LTE tolerance $\varepsilon$ and suggests that TR-FDI- $n$ and TR-TSA- $n$ have comparable economy and efficiency. TR-FDI-01 and TR-FDI-02 even appear to have worse economy when $\varepsilon<10^{-4}$. This performance reduction comes from instability due to large step size changes at early times and can be avoided by setting a limit on time step size growth, e.g., $\Delta t_{k+1} \leq 1.5 \Delta t_{k}$ [33].

Figure 8(b) again recasts the results in terms of the accumulated time discretization error estimate $E_{g}$ using (2.5). The second order accurate TR-FDI- $n$ is actually more economical and more efficient than the first order accurate TR-TSA- $n$ when compared on such a basis.

4. Viscoelastic stretching filament. Transient viscoelastic free surface flow calculations are performed by solving simultaneously the system of fully coupled equations (Petrov-Galerkin FEM) for momentum, continuity, conformation, interpolated velocity gradient, and mesh generation $[7,43]$. Several added features to the fluid flow problem are expected to increase the importance of the integrator's accuracy and stability: (1) the elastic stress conservation equation is hyperbolic, (2) viscoelasticity and the dynamic boundaries add new timescales to stiffen the problem, and (3) the coupling between space and time discretizations is strengthened in the arbitrary Lagrangian-Eulerian (ALE) formulation for solving free surface problems.

Modeling and numerical considerations. We use a viscoelastic constitutive law derived from the finitely extensible nonlinear elastic dumbbell model with Peterlin's closure approximation (FENE-P) [9]. In our FEM code, this model is implemented within a conformation tensor framework $[34,44]$ and reads 

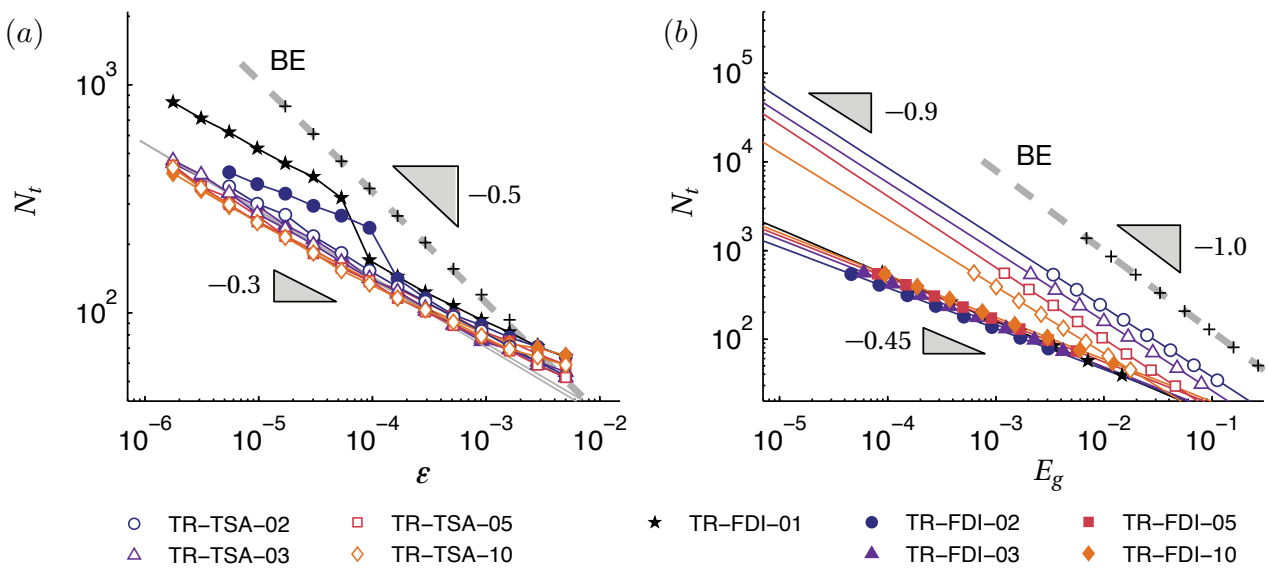

FIG. 8. Number of time steps $N_{t}$ to achieve $t \geq 200$ with respect to (a) LTE tolerance $\varepsilon$ and (b) accumulated time discretization error $E_{g}$ (as determined at $t=t_{a}$ ).

$$
\begin{aligned}
\frac{\partial \mathbf{M}}{\partial t}+\mathbf{v} \cdot \nabla \mathbf{M}-\mathbf{M} \cdot \nabla \mathbf{v}-\nabla \mathbf{v}^{T} \cdot \mathbf{M} & =-\frac{1}{\lambda}\left(\frac{b-1}{b-\frac{\operatorname{tr} \mathbf{M}}{3}} \mathbf{M}-\mathbf{I}\right), \\
\boldsymbol{\tau}_{e} & =\frac{\eta_{e}}{\lambda}(\mathbf{M}-\mathbf{I}) \cdot \mathbf{I} \frac{b-1}{b-\frac{\operatorname{tr} \mathbf{M}}{3}},
\end{aligned}
$$

where $\mathbf{M}$ is a dimensionless conformation tensor embodying the microstructural details of the liquid and $\boldsymbol{\tau}_{e}$ is the elastic stress. The relaxation time $\lambda$, polymer viscosity $\eta_{e}$, and extensibility parameter $b$ are the viscoelastic material parameters.

Following [43], we use the discrete elastico-viscous split stress-traceless interpolated velocity gradient/streamline upwind Petrov-Galerkin (DEVSS-TG/SUPG) method. SUPG [12] partially addresses the instability associated with the hyperbolic conservation equation (4.1). The DEVSS-TG formulation requires simultaneous solution of an additional unknown, the traceless interpolated velocity gradient $\mathbf{L}$

$$
\mathbf{L}=\nabla \mathbf{v}-(\nabla \cdot \mathbf{v}) \mathbf{I} / \operatorname{tr} \mathbf{I}
$$

which replaces $\nabla \mathbf{v}$ in (4.1) and modifies (3.3) after adding the elastic stress $\boldsymbol{\tau}_{e}$,

$$
\mathbf{T}=-p \mathbf{I}+\eta_{s}\left[\mathbf{L}+\mathbf{L}^{\mathrm{T}}\right]+\eta_{a}\left[\nabla \mathbf{v}+(\nabla \mathbf{v})^{\mathrm{T}}-\mathbf{L}-\mathbf{L}^{\mathrm{T}}\right]+\boldsymbol{\tau}_{e},
$$

where $\eta_{a}$ is a numerical parameter. The interpolated velocity gradient equation (4.3) is not dynamic and therefore is excluded from the LTE estimator for adaptive time stepping. The scalar components of $\mathbf{M}$ and $\mathbf{L}$ are supported on $Q_{1}$, while the velocity and pressure variables are supported as before on $Q_{2} P_{-1}$.

In standard FEM, the set of equations posed in the physical domain $\mathbf{x}$ are transformed to an equivalent set posed in a logical or reference domain $\boldsymbol{\xi}$ by the mapping $\mathbf{x}=\mathbf{x}(\boldsymbol{\xi})$. Deforming domains are thus easily handled by treating the mesh positions $\mathbf{x}$ as an additional set of unknowns (supported on $Q_{2}$ ) governed by $[14,16]$

$$
\mathbf{0}=\nabla \cdot \tilde{\mathbf{D}} \cdot \nabla \boldsymbol{\xi}
$$

where $\tilde{\mathbf{D}}$ controls the distribution of elements throughout the domain. The boundary locations and velocities are governed by independent conditions on the externally 
forced moving walls, and by the coupled normal stress balance and kinematic interface conditions on the free surface,

$$
\begin{aligned}
0 & =\mathbf{n} \cdot(\mathbf{v}-\dot{\mathbf{x}}), \\
\mathbf{n} \cdot \mathbf{T} & =\left(-p_{\mathrm{amb}}+\kappa \sigma\right) \mathbf{n},
\end{aligned}
$$

where $\mathbf{n}$ is the outward pointing boundary normal, $\kappa$ is the boundary curvature, $\sigma$ is the interfacial tension, and $p_{\text {amb }}$ is the ambient pressure. The fluid's density, viscosity, and interfacial tension with the surroundings set two time scales: the viscous and capillary time scales. Elastic relaxation and forced boundary motions are additional time scales that potentially stiffen the problem.

In the ALE formulation, mesh locations neither are fixed in space nor convect with $\mathbf{v}$ so that conservation equations for the general quantity $\boldsymbol{\Phi}$ posed in a fixed frame must be transformed to account for the "mesh velocity" $\dot{\mathbf{x}}[16,6]$

$$
\frac{\partial \Phi}{\partial t}=\dot{\Phi}-\dot{\mathbf{x}} \cdot \nabla \boldsymbol{\Phi}
$$

with $\dot{\boldsymbol{\Phi}}$ the time rate of change of the nodal value. As a result, the space-time coupling is strengthened through the convective terms of the conservation equations, which require not only the velocity field but also the time derivative of the mesh field to compute. The mesh positions are excluded from the LTE estimator because (4.5) is not dynamic, even though $\dot{\mathbf{x}}$ are computed for (4.8).

The filament stretching problem is solved as a $2 \mathrm{D}$ axisymmetric problem. The domain is discretized into $12 \times 640$ quadrilateral elements (radial $\times$ axial), where the axial refinement enables good resolution of the elongating domain. The dimensionless initial time step size is set to $\Delta t_{0}=10^{-6}$.

Problem description. An initially quiescent cylindrical liquid bridge with height $L_{0}$ and radius $R_{0}$ is held between two flat plates and stretched as the plates are separated with velocity $U_{c}$. The plates are stopped beyond a critical separation, whereupon the unstable liquid filament undergoes capillary thinning. This process exemplifies capillary break-up extensional rheometry (CaBER), an experimental technique for characterizing the extensional rheology of non-Newtonian liquids. Gravity is neglected and the solid-liquid-gas contact line is pinned to the plates at $R_{0}$. The relevant physical parameters and time scales are defined in Table 1.

The dimensionless polymer extensibility parameter is set to $b=100$, a value that makes the solution moderately challenging and is typical of flexible polymer solutions. The remaining parameters are chosen so that the elastic, viscous, and capillary time scales are all equal; these can interchangeably be used to define a dimensionless time. The top plate is accelerated to constant nominal velocity at time $t_{d}=0.6$ and decelerated to rest at $t_{s}=2.6$, such that the final plate separation is $L_{f}=3 L_{0}$ (Appendix $\mathrm{C}$ ). This system does not tend to a steady state, and the integration is terminated at $t_{\text {end }}=10$ when the thinning filament neck radius has reduced to $r_{\text {mid }} / R_{0}<0.1$. While our choice of parameters does not stiffen the system, the shocks in the boundary velocity during plate acceleration induces TR stall.

The quantity sought in CaBER is the extensional viscosity $\eta_{E}=\left(T_{33}-T_{11}\right) / \dot{\epsilon}$, where $\dot{\epsilon}$ is the axial extension rate in the fluid and $T_{33}-T_{11}$ is the axial-radial normal stress difference. A meaningful and convenient solution metric is therefore the midfilament normal stress difference at the free surface, denoted $\tau_{\Delta}$; it is a combination of all field variables except pressure, which is subtracted out by definition. The 
TABLE 1

Parameters and quantities relevant to the viscoelastic liquid filament problem.

\begin{tabular}{rll}
\hline Symbol & Definition & Description \\
\hline$\rho$ & & liquid density \\
$\sigma$ & & interfacial tension at the free surface \\
$\eta_{s}$ & & solvent viscosity \\
$\eta_{e}$ & & polymer viscosity \\
$\eta_{0}$ & $\eta_{s}+\eta_{e}$ & zero-shear viscosity \\
$\lambda$ & & elastic time scale \\
$b$ & & dimensionless polymer extensibility parameter \\
$U_{c}$ & & nominal stretching velocity \\
$\bar{t}_{v}$ & $\eta_{0} R_{0} / \sigma$ & viscous time scale \\
$\bar{t}_{c}$ & $\sqrt{\rho R_{0}^{3} / \sigma}$ & capillary time scale \\
$\bar{t}_{e}$ & $R_{0} / U_{c}$ & stretching time scale \\
$\mathrm{Ca}$ & $U_{c} \eta_{0} / \sigma=\bar{t}_{v} / \bar{t}_{e}$ & dimensionless capillary number \\
\hline
\end{tabular}
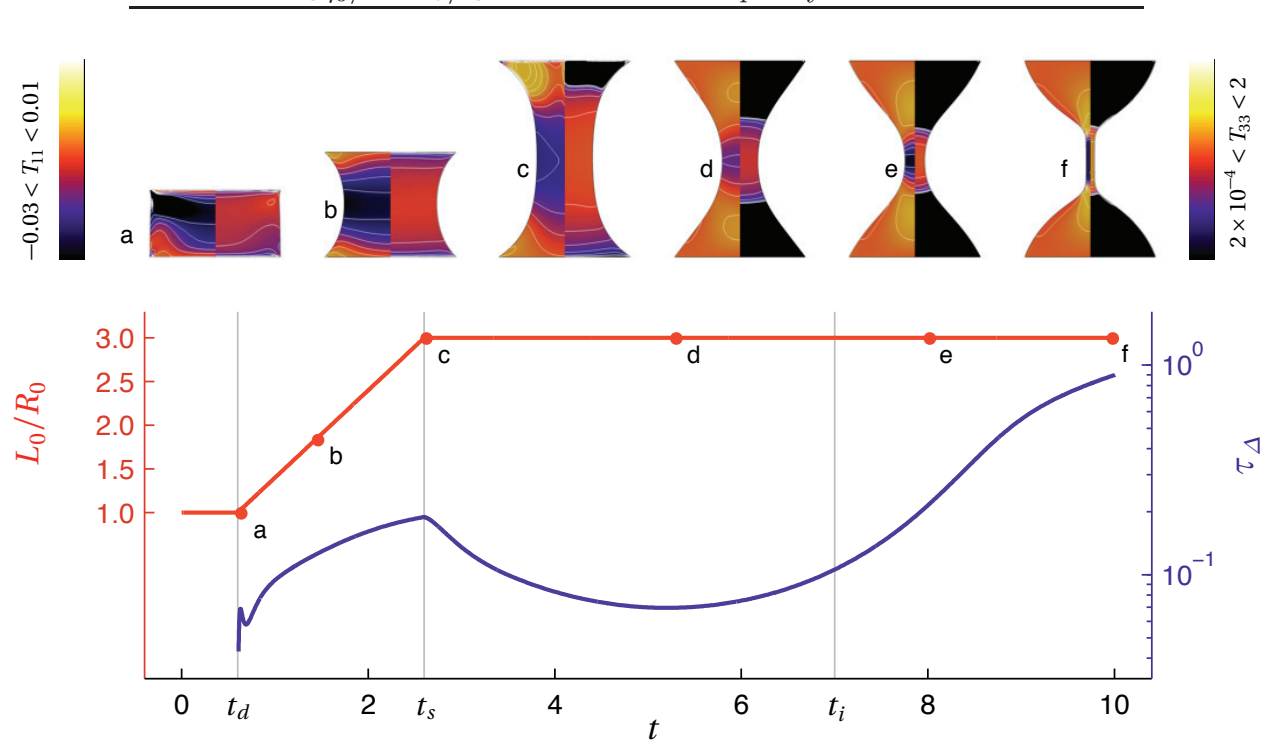

FIG. 9. Evolution of the relative plate separation $L_{0} / R_{0}$ and midfilament free surface normal stress difference $\tau_{\Delta}$. Top: representative snapshots (computed by TR-FDI-01) of the normal stress components. Contours of $T_{33}$ are on a log scale.

evolution of $\tau_{\Delta}$ is shown in Figure 9 along with the plate separation evolution and representative snapshots of the normal stress components (computed by TR-FDI-01). When the plate comes to rest at $t_{s}$, the behavior of $\tau_{\Delta}$ changes qualitatively as the dynamics transition from forced stretching to self-thinning.

Results. TR appears more susceptible to stall for this problem and fails to reach termination regardless of initial time step size. Accuracy analysis is carried out as before using the midfilament free surface normal stress $\tau_{\Delta}$ as the solution metric. The accumulated error estimate $E_{g}=\left(\tau_{\Delta}-\tau_{\Delta, 0}\right) / \tau_{\Delta, 0}$ is plotted in Figure 10 with respect to LTE tolerance $\varepsilon$ at two times $t_{s}=2.6$ and $t_{i}=7.0$. Because TR reference solutions are unavailable, the asymptotic solution estimate $\tau_{\Delta, 0}$ is obtained by extrapolating the TR-FDI- $n$ results (averaged over the values for all $n$ considered).

$\mathrm{BE}$ is verified to be first order accurate, TR-TSA- $n$ exhibits diminished order of accuracy, and TR-FDI- $n$ remains second order accurate. The added challenges associated with viscoelasticity and the free surface do not appear to significantly 

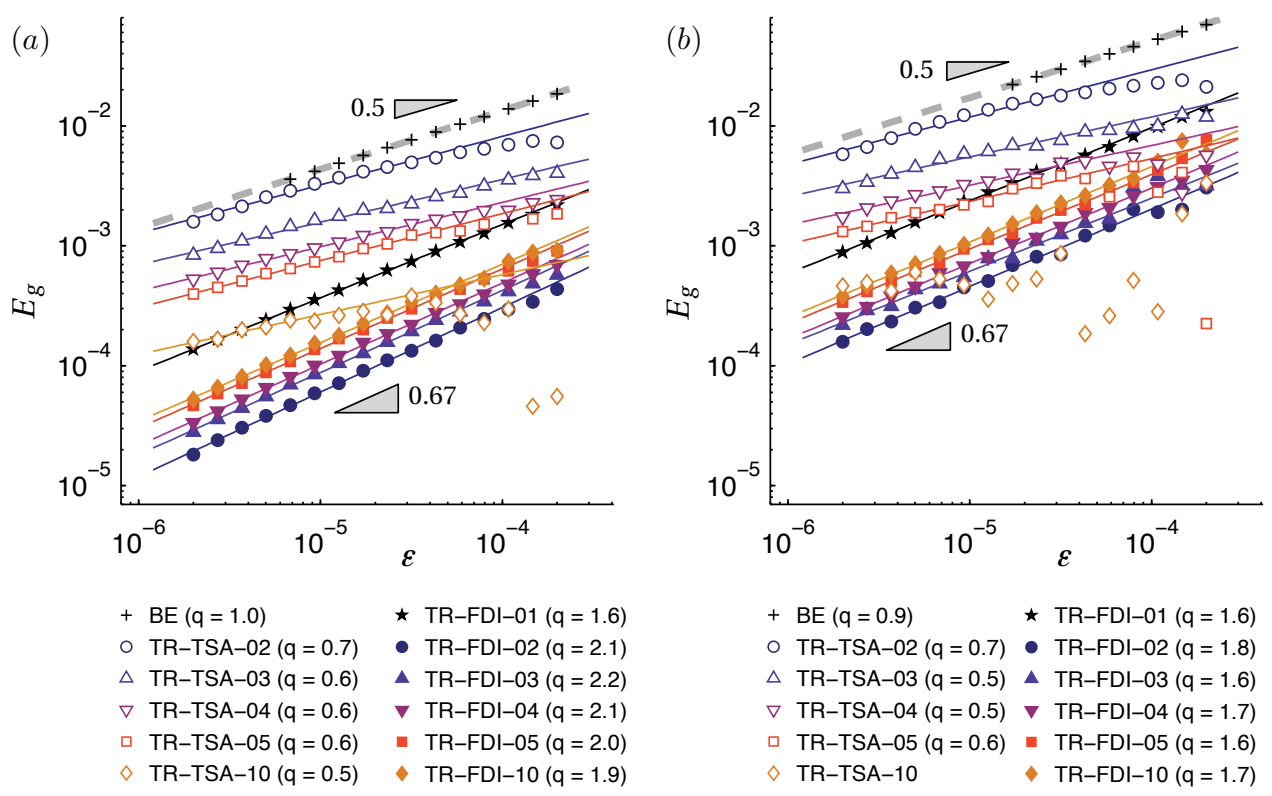

FIG. 10. Accumulated time discretization error estimate $E_{g}=\left(\tau_{\Delta}-\tau_{\Delta, 0}\right) / \tau_{\Delta, 0}$ with respect to LTE tolerance $\varepsilon$ at dimensionless times (a) $t_{s}=2.6$ and (b) $t_{s}=7.0$.

influence the accuracy characteristics of either stabilization method. Compared to previous problems, the accuracy of TR-TSA- $n$ relative to BE actually appear slightly improved; this may be related to the increased importance of reducing spurious diffusion when solving the hyperbolic conformation equation. The errors of TR-TSA-10 are anomalously low, but with erratic dependence on $\varepsilon$; TR-FDI- $n$ with large $n$ have more predictable errors and appear to better reflect the pure TR limit.

Typical step size progressions are shown in Figure 11 for fixed value of $\varepsilon$. With TR, adaptive time stepping is always compromised and usually stalls. With all other methods, adaptive time stepping responds as expected based on the physics: $\Delta t$ increases during the initial latency period, decreases sharply to resolve the accelerations at $t_{d}$ and $t_{s}$, and recovers to larger values in the absence of forced accelerations.

The number of steps $N_{t}$ taken to reach termination at $t_{\text {end }}=10$ is plotted in Figure 12 (a) as a function of $\varepsilon$. Though TR-TSA- $n$ is still computationally more economical and efficient than BE, its efficiency is worsened in comparison to previous problems ( $\chi$ increases from 0.3 to 0.4 ). Moreover, its economy worsens measurably as $n$ decreases. The economy of TR-FDI- $n$ does not depend as strongly on $n$, and its efficiency does not appear to be degraded $(\chi=0.3)$. Thus, in contrast to previous problems, TR-FDI- $n$ is clearly more economical and efficient than TR-TSA- $n$, even on the basis of local error tolerance. Figure 12(b) recasts the results in terms of the accumulated time discretization error up to time $t=t_{s}$. The performance advantage of TR-FDI- $n$ is again better emphasized on this basis.

Despite taking significantly fewer steps, Figure 13(a) shows that TR-TSA- $n$ and TR-FDI- $n$ do not take correspondingly less time to execute than BE on the basis of LTE tolerance $\varepsilon$. The extra execution time is spent on step rejections, where the LTE fails to meet the tolerance $\left(e_{k+1} / \varepsilon>1.5\right.$ in our code). Steps are typically rejected when the adaptive algorithm fails to anticipate abrupt solution changes, as by 


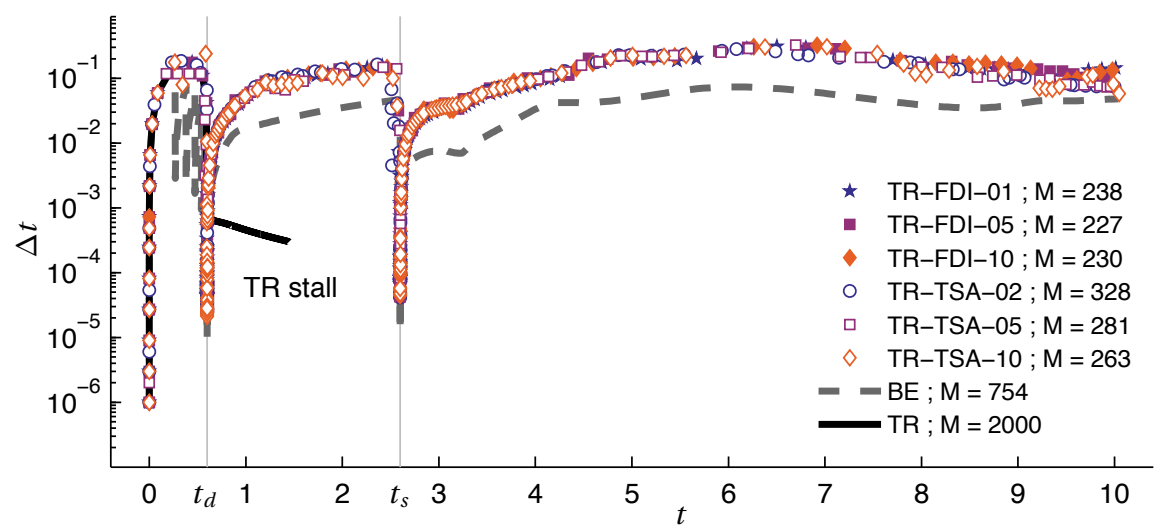

FIG. 11. Time step size progression during viscoelastic filament stretching/thinning problem for $\varepsilon=1.47 \times 10^{-4}$. The light vertical lines indicate times of maximum acceleration where time step sizes are small. The legend indicates the total number of time steps $M$ taken to achieve $t \geq t_{\mathrm{end}}$.
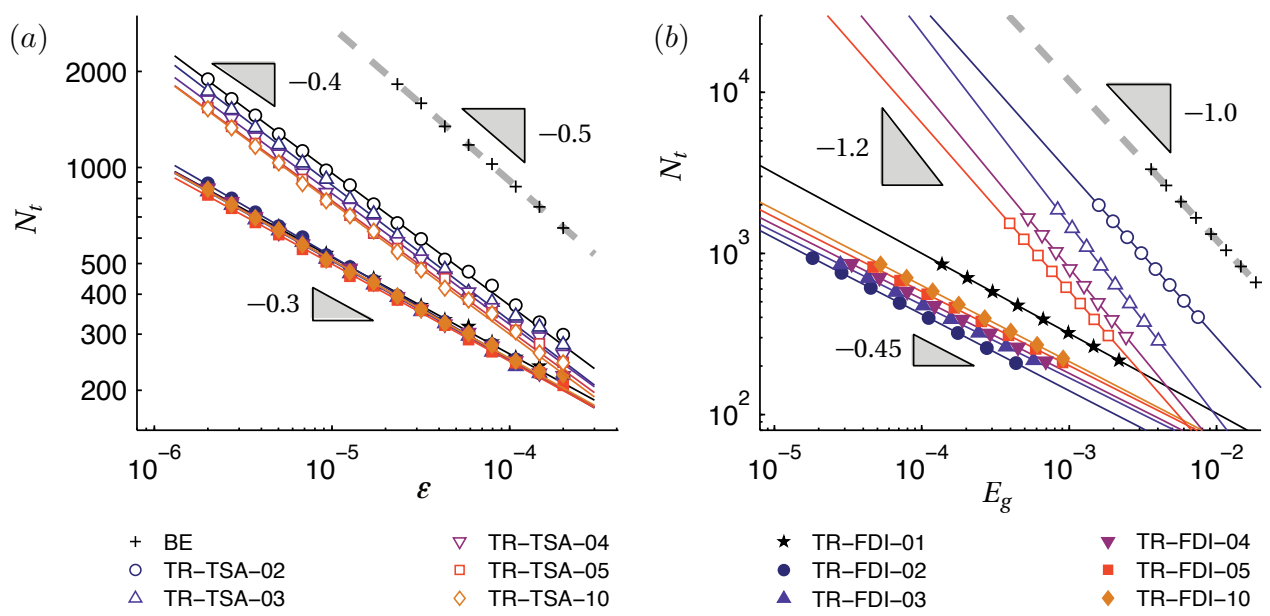

FIG. 12. Number of time steps $N_{t}$ required to reach $t_{\mathrm{end}}=10$ with respect to (a) LTE tolerance $\varepsilon$ and (b) approximate tolerance on $E_{g}$ (as determined at $t=t_{s}$ ).

external forcing. Figures 13(a) and 12(a) together indicate that TR-FDI- $n$ is rejecting more steps than TR-TSA- $n$. The incongruously short execution times for TR-FDI-01 indicate comparatively fewer step rejections, especially with stricter tolerance. On the basis of $E_{g}$, TR-FDI- $n$ still generally maintains a decisive advantage compared to TR-TSA- $n$ and BE (Figure 13(b)).

Figure 14(a) compares the step rejection frequency for fixed $\varepsilon$ as a function of the interval $n$ for both TR-FDI- $n$ and TR-TSA- $n$. The same comparison is shown for the lid-driven cavity, where step rejection frequency is negligible because the driving lid acceleration is sufficiently gradual. TR-TSA- $n$ suffers more step rejections as $n$ decreases, indicating that TSA interventions are corrupting the LTE-based adaptive stepping algorithm: TSA shifts and modifies the solutions and derivatives such that both the AB2 predictor and the LTE are inconsistent with those at previous steps. 



FIG. 13. Execution times as function of (a) $\varepsilon$ and (b) $E_{g}$ (as determined at $t=t_{s}$ ).
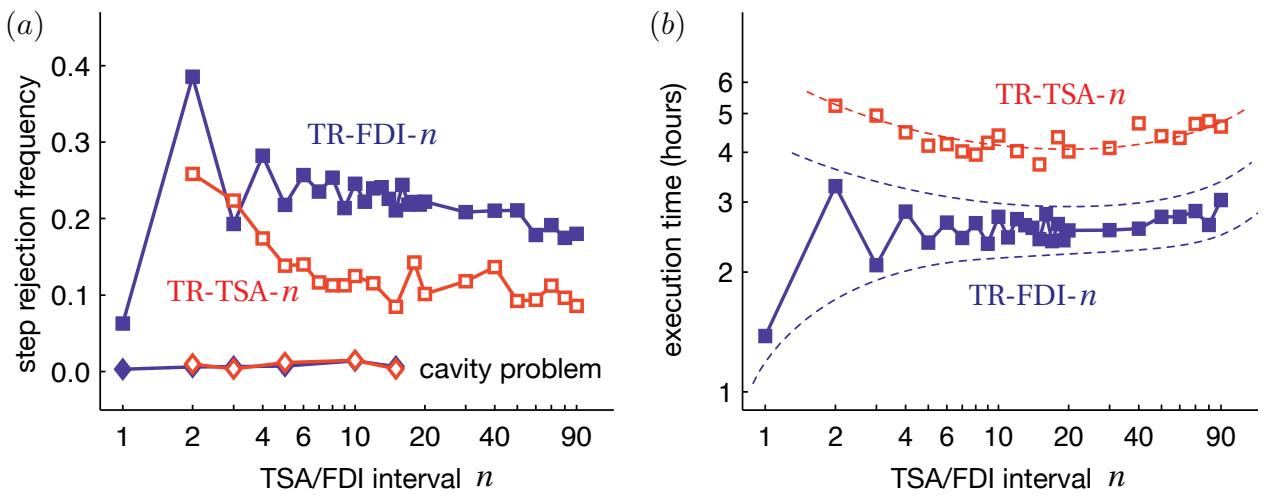

FIG. 14. (a) Step rejection frequency, defined as the ratio of rejected to accepted steps. Execution times with dashed lines to highlight trends. Comparisons at fixed $\varepsilon=10^{-5}$.

Total execution times with respect to $n$ are plotted in Figure 14(b). The trend for TR-TSA- $n$ suggests an optimal value of $n \approx 10$, which is consistent with the default choice in [37]; however, a good choice of $n$ depends also on the LTE tolerance $\varepsilon$ [31]. Specifically, the optimal $n$ is that above which a corrupted LTE analysis exacerbates step rejection and below which the underlying TR integrator spends more steps in a ringing-induced stall. It is not possible to determine a universally optimal $n$ because TR's instability and the algorithm's susceptibility to LTE corruption are problemdependent. A strategy for determining $n$ on the fly is discussed in [31] but appears to be based on a priori knowledge of the time scales present in the system.

For TR-FDI- $n$ with $n<20$, the step rejection frequency depends strongly on whether $n$ is even or odd. The adaptive step algorithm depends on consistent expressions of $\dot{y}_{k-1}$ and $\dot{y}_{k}$, but only the latter is modified following an FDI. Two steps after an FDI, only the earlier derivative $\dot{y}_{k}$ has been modified and $\dot{y}_{k+1}$ remains a TR derivative. TR-FDI-02 switches the derivative representation every step so that the LTE estimator and AB2 predictor are always inconsistent; it executes in the longest time with the most rejected steps. TR-FDI-01 modifies the derivative at every step, 
and therefore the LTE estimator and AB2 predictor are always based on consistent derivatives; it appears to be the best case with the fastest execution time. Other odd$n$ methods are similarly less susceptible to FDIs, suffering fewer rejected steps than even- $n$ methods. A good compromise between accuracy and excessive step rejection appears to be $n=3$ or $n=5$.

5. Conclusions. We have presented a simple new scheme (TR-FDI) to stabilize the ringing-induced stall during adaptive step TR. TR-FDI suppresses the asymptotically oscillatory behavior of TR by intermittently replacing the last computed TR-derivative of the solution with the retrospectively computed second order backward difference. It is similar to the time step averaging method (TR-TSA), which uses shifted first order finite differences instead; both are trivial to implement in an existing TR-based code with virtually no added computational cost, but TR-FDI is more accurate with better computational economy and efficiency.

TR-FDI is $A$-unstable and therefore $L$-unstable; however, it is stiffly stable and does effectively eliminate the ringing instability to prevent adaptive step stall. On the basis of LTE tolerance, it was shown to be comparable in computational economy and efficiency to TR-TSA, BDF2, and TR-BDF2 when solving the pure diffusion and pure oscillatory scalar ODE test problems. Though equally or more economical and efficient than TR-FDI, TR-TSA suffers reduced order accuracy, whereas TRFDI remains second order accurate. Thus, when compared on the basis of actually accumulated time discretization errors, TR-FDI is the most economical and efficient method.

When applied to fluid flow problems - the transient lid-driven cavity and the viscoelastic stretching/thinning liquid filament-TR-FDI was again verified to preserve second order accuracy and to exhibit better economy and efficiency. In contrast to the lid-driven cavity problem, the viscoelastic filament problem was less tolerant to TR-TSA, as demonstrated by degraded economy and efficiency even on the basis of LTE tolerance. This is attributed to tighter space-time coupling by ALE and the hyperbolic nature of the viscoelasticity equation, both of which make the problem less forgiving to time-discretization errors and spurious damping.

Though demonstrated here with an FEM/ALE method-of-lines, the TR-FDI scheme can be applied easily to any TR-based numerical PDE solver. Additional insight into the stability of TR-FDI may be gained by studying stiffer problems, more strongly convection-dominated problems, or problems with cyclical solutions in time. Also, it may be possible to reduce excessive step rejection and improve execution times by correcting the LTE estimator following an FDI; a more complete study on the error analysis of TR-FDI is thus currently under way. Another question is whether the mesh variables should be included in the LTE estimator when their time derivatives are implicitly considered in the nonlinear solution, as in ALE.

In summary, we have demonstrated that TR-FDI is generally more efficient and robust than existing stabilization methods of comparable complexity. Furthermore, it preserves second order accuracy, is easy to implement in existing TR-based codes, and adds negligible computational cost.

Appendix A. TR-TSA. The stabilized TR by time step averaging (TSA) [31] is outlined here for the scalar variable $y$. The TSA operation described below is applied periodically every $n>1$ time steps to yield the TR-TSA- $n$. Following a successful TR step, the consecutive solutions are $y_{k-1}, y_{k}, y_{k+1}$, and their derivatives are $\dot{y}_{k-1}$, $\dot{y}_{k}, \dot{y}_{k+1}$ at times $t_{k-1}, t_{k}, t_{k+1}$. The time step sizes are denoted $\Delta t_{k}=t_{k}-t_{k-1}$ and $\Delta t_{k+1}=t_{k+1}-t_{k}$. Temporary variables $t_{*}=t_{k}, t_{* *}=t_{k+1}, y_{*}=y_{k}, \dot{y}_{*}=\dot{y}_{k}$, 
$y_{* *}=y_{k+1}$, and $\dot{y}_{* *}=\dot{y}_{k+1}$ are defined in anticipation of the time shifts at steps $k$ and $k+1$. Following the formulations of [37], we define the "TR update" $d_{k}$

$$
y_{* *}=y_{*}+\Delta t_{k+1} d_{k},
$$

which by (1.2) can also be thought of as the average derivative at the shifted $t_{k}$ :

$$
d_{k}=\frac{1}{2}\left(\dot{y}_{*}+\dot{y}_{* *}\right)
$$

Next, the time steps are shifted to $t_{k}=t_{k-1}+\Delta t_{k} / 2$ and $t_{k+1}=t_{*}+\Delta t_{k+1} / 2$, and following [37], the shifted solutions and time derivatives are defined as

$$
\begin{aligned}
& y_{k}=\frac{1}{2}\left(y_{k-1}+y_{*}\right), \dot{y}_{k}=\frac{1}{2}\left(\dot{y}_{k-1}+\dot{y}_{*}\right), \\
& y_{k+1}=y_{*}+\frac{1}{2} \Delta t_{k+1} d_{k}, \quad \dot{y}_{k+1}=d_{k} .
\end{aligned}
$$

It is clear that (A.3) are averages, while (A.4) may appear at first glance to be an application of the second order accurate TR with half-step advance (the derivative is still clearly an average). Upon rearrangement, (A.4) becomes

$$
y_{k+1}=\frac{1}{2}\left(y_{*}+y_{* *}\right), \quad \dot{y}_{k+1}=\frac{y_{* *}-y_{*}}{\Delta t_{k+1}},
$$

so that the shifted $y_{k+1}$ is also clearly an average, and $\dot{y}_{k+1}$ is written explicitly as a two-point backward difference with first order accuracy.

Appendix B. Growth factor for TR-FDI-01. The numerical solution to an ODE can be expressed as a cumulative growth of the previous solution with step growth factor $\xi$ :

$$
y_{k-j}=\xi^{-j} y_{k} .
$$

For the prototypical scalar problem $\dot{y}=\gamma y$, the TR solution has an explicit form

$$
y_{k+1}=\frac{2 y_{k}+\Delta t \dot{y}_{k}}{2-\gamma \Delta t} \text {. }
$$

Together, (B.1) and (B.2) give the growth factor for TR as (1.6). In TR-FDI-01, $\dot{y}_{k}$ is replaced by the backward difference formula (1.7) before the $k+1$ solution is calculated. The backward difference formula for uniform step size $\Delta t$ is

$$
\dot{y}_{k}^{\mathrm{bd}}=\frac{3 y_{k}-4 y_{k-1}+y_{k-2}}{2 \Delta t} .
$$

Substituting (B.3) into (B.2), rewriting all terms using (B.1), and defining $\psi=\gamma \Delta t$,

$$
\xi y_{k}=\frac{2 y_{k}+\frac{3 y_{k}-4 \xi^{-1} y_{k}+\xi^{-2} y_{k}}{2}}{2-\psi},
$$

a third order polynomial that rearranges to $(2.2)$, and whose $\ell$ th root is found to be

$$
\xi_{\ell}=\frac{-14+\mathcal{A}_{\ell}(1+24 \psi) \mathcal{B}^{-1 / 3}+\overline{\mathcal{A}}_{\ell} \mathcal{B}^{1 / 3}}{12 \psi-24},
$$

where $\overline{\mathcal{A}}_{\ell}$ is the complex conjugate of $\mathcal{A}_{\ell}$, and

$$
\begin{aligned}
\mathcal{B} & =-54 \psi^{2}+36 \psi-55+6(\psi-2) \sqrt{3\left(27 \psi^{2}+16 \psi+7\right)}, \\
\mathcal{A}_{1} & =2 \\
\mathcal{A}_{2} & =-1-i \sqrt{3} \\
\mathcal{A}_{3} & =-1+i \sqrt{3} .
\end{aligned}
$$

Copyright $@$ by SIAM. Unauthorized reproduction of this article is prohibited. 
Similarly, the growth factor expression for TR-FDI- $n$ reaches back one earlier time step for every increase in $n$ so that the characteristic equations for TR-FDI- $n$ are polynomials of order $n+2$.

Appendix C. Ramp function for filament stretching. For the filament stretching problem of section 4 , the plate axial position $z(t)$ and separation velocity $v(t)=\dot{z}(t)$ are imposed independently as boundary conditions on the mesh and momentum equations, respectively:

$$
\begin{gathered}
(\mathrm{C} .1) \quad z(t)=\frac{L_{0}+L_{f}}{2}+\frac{U_{c}^{2}}{4 a} \log \left[\frac{\cosh \left[\frac{2 a}{U_{c}}\left(t-t_{d}\right)\right]}{\cosh \left[\frac{2 a}{U_{c}}\left(t-t_{d}-\frac{L_{f}-L_{0}}{U_{c}}\right)\right]}\right], \\
\left(\text { C.2 } v(t)=\dot{z}(t)=\frac{U_{c}}{2}\left\{\tanh \left[\frac{2 a}{U_{c}}\left(t-t_{d}\right)\right]-\tanh \left[\frac{2 a}{U_{c}}\left(t-t_{d}-\frac{L_{f}-L_{0}}{U_{c}}\right)\right]\right\},\right.
\end{gathered}
$$

where $L_{0}$ is the initial plate position, $L_{f}$ is the final plate position, $U_{c}$ is the plateau velocity, and $t_{d}$ marks the time at maximum acceleration $a$.

Acknowledgments. J. Alex Lee and Matteo Pasquali thank Professors Dan Sorensen, Mark Embree, and Tim Warburton for their feedback and discussion.

\section{REFERENCES}

[1] A. Abedijaberi, G. Bhatara, E. S. G Shaqfeh, and B. Khomami, A computational study of the influence of viscoelasticity on the interfacial dynamics of dip coating flow, J. NonNewtonian Fluid Mech., 166 (2011), pp. 614-627.

[2] N. L. C. Arruda, R. C. Almeida, R. S. Silva, and S. M. C. Malta, Avoiding spurious modes of time discretized operators in transport problems, Int. J. Numer. Methods Biomed. Eng., 26 (2010), pp. 609-627.

[3] U. M. Ascher and L. R. Petzold, Computer Methods for Ordinary Differential Equations and Differential-Algebraic Equations, SIAM, Philadelphia, 1998.

[4] R. E. Bank, W. M. Coughran, W. Fichtner, E. H. Grosse, D. J. Rose, and R. K. Smith, Transient simulation of silicon devices and circuits, IEEE Trans. Electron Dev., 4 (1985), pp. $436-451$.

[5] P. P. Bhat, S. Appathurai, M. Harris, M. Pasquali, G. H. McKinley, and O. A. Basaran, Formation of beads-on-a-string structures during break-up of viscoelastic filaments, Nature Phys., 6 (2010), pp. 625-631.

[6] P. P. Bhat, O. A. Basaran, And M. Pasquali, Dynamics of viscoelastic liquid filaments: Low capillary number flows, J. Non-Newtonian Fluid Mech., 150 (2008), pp. 211-225.

[7] P. P. Bhat, M. Pasquali, and O. A. Basaran, Beads-on-string formation during filament pinch-off: Dynamics with the PTT model for non-affine motion, J. Non-Newtonian Fluid Mech., 159 (2009), pp. 64-71.

[8] G. Bhatara, E. S. G. Shaqfeh, and B. Khomami, The influence of polymer concentration and chain architecture on free surface displacement flows of polymeric fluids, J. Rheol., 49 (2005), 929.

[9] R. B. Bird, P. J. Dotson, And N. L. Johnson, Polymer-solution rheology based on a finitely extensible bead-spring chain model, J. Non-Newtonian Fluid Mech., 7 (1980), pp. 213-235.

[10] D. Britz and O. Østerby, Some numerical investigations of the stability of electrochemical digital simulation, particularly as affected by first-order homogeneous reactions, J. Electroanal. Chem., 368 (1994), pp. 143-147.

[11] D. Britz, O. Østerby, And J. Strutwolf, Damping of Crank-Nicolson error oscillations, Comput. Bio. Chem., 27 (2003), pp. 253-263.

[12] A. N. Brooks and T. J. R. Hughes, Streamline Upwind/Petrov-Galerkin formulations for convection dominated flows with particular emphasis on the incompressible Navier-Stokes equations, Comput. Methods Appl. Mech. Engrg., 32 (1982), pp. 199-259.

[13] R. A. Cairncross, P. R. Schunk, T. Baer, R. R. Rao, and P. Sackinger, A finite element method for free surface flows of incompressible fluids in three dimensions. Part I. Boundary fitted mesh motion, Internat. J. Numer. Methods Fluids, 33 (2000), pp. 375-403.

Copyright $@$ by SIAM. Unauthorized reproduction of this article is prohibited. 
[14] M. S. Carvalho and L. E. Scriven, Flows in forward deformable roll coating gaps: Comparison between spring and plane-strain models of roll cover, J. Comput. Phys., 138 (1997), pp. 449-479.

[15] J. CASH, Split linear multistep methods for the numerical integration of stiff differential systems, Numer. Math., 42 (1983), pp. 299-310.

[16] K. N. Christodoulou And L. E. Scriven, Discretization of viscous free surface flows and other free boundary problems, J. Comput. Phys., 99 (1992), pp. 39-55.

[17] J. Chung And G. M. Hulbert, A time integration algorithm for structural dynamics with improved numerical dissipation: The generalized- $\alpha$ method, J. Appl. Mech., 60 (1993), p. 371.

[18] C. H. Cooke, A characterization of stiffly stable linear multistep methods, Internat. J. Numer. Methods Engrg., 7 (1973), pp. 117-124.

[19] J. CRANK AND P. NiCOlson, A practical method for numerical evaluation of solutions of partial differential equations of the heat-conduction type, Proc. Cambridge Philosophical Soc., 43 (1947), pp. 50-67.

[20] C. F. Curtiss and J. O. Hirschfelder, Integration of stiff equations, Proc. Nat. Acad. Sci. USA, 38 (1952), pp. 235-243.

[21] G. G. DAhlQuist, A special stability problem for linear multistep methods, BIT, 3 (1963), pp. 27-43.

[22] W. G. Dettmer And D. Perić, An analysis of the time integration algorithms for the finite element solutions of incompressible Navier-Stokes equations based on a stabilised formulation, Comput. Methods Appl. Mech. Engrg., 192 (2003), pp. 1177-1226.

[23] S. Dharmaraja, Y. Wang, and G. Strang, Optimal stability for trapezoidal-backward difference split-steps, IMA J. Numer. Anal., 30 (2010), pp. 141-148.

[24] H. Elman, M. Mihajlovic, and D. J. Silvester, Fast iterative solvers for buoyancy driven flow problems, J. Comput. Phys., 230 (2011).

[25] H. Elman, D. J. Silvester, And A. Wathen, Finite Elements and Fast Iterative Solvers: With Applications in Incompressible Fluid Dynamics, 2nd ed., Oxford University Press, New York, 2014.

[26] S. Erlicher, L. Bonaventura, and O. S. Bursi, The analysis of the generalized- $\alpha$ method for non-linear dynamic problems, Comput. Mech., 28 (2002), pp. 83-104.

[27] R. Ganesan and N. J. Salamon, A self-optimizing single-step algorithm for the numerical integration of parabolic equations, Comput. Methods Appl. Mech. Engrg., 125 (1995), pp. 109121.

[28] C. W. GEAR, The automatic integration of stiff ordinary differential equations, in Proceedings of the IFIPS Conference, 1968, pp. A81-A85.

[29] C. W. GEar, Numerical Initial Value Problems in Ordinary Differential Equations, PrenticeHall, Englewood Cliffs, NJ, 1971.

[30] R. GLOwinski, Viscous flow simulation by finite element methods and related numerical techniques, in Progress and Supercomputing in Computational Fluid Dynamics, Progr. Sci. Compute. 6, 1985, pp. 173-210.

[31] P. M. Gresho, D. F. Griffiths, and D. J. Silvester, Adaptive time-stepping for incompressible flow part I: Scalar advection-diffusion, SIAM J. Sci. Comput., 30 (2008), pp. 2018-2054.

[32] P. M. Gresho, R. L. Lee, And R. L. Sani, Recent advances in Numerical Methods in Fluids, Pineridge Press, Whiting, NJ, 1980.

[33] P. M. Gresho, R. L. Sani, And M. S. Engelman, Incompressible Flow and the Finite Element Method: Advection-Diffusion and Isothermal Laminar Flow, Wiley, New York, 1998.

[34] M. Grmela and P. J. Carreau, Conformation tensor rheological models, J. Non-Newtonian Fluid Mech., 23 (1987), pp. 271-294.

[35] M. E. Hosea And L. F. Shampine, Analysis and implementation of TR-BDF2, Appl. Numer. Math., 20 (1996), pp. 21-37.

[36] G. M. Hulbert And J. Chung, The unimportance of the spurious root of time integration algorithms for structural dynamics, Commun. Numer. Methods Engrg., 10 (2005), pp. 591597.

[37] D. A. Kay, P. M. Gresho, D. F. Griffiths, and D. J. Silvester, Adaptive time-stepping for incompressible flow Part II: Navier-Stokes equations, SIAM J. Sci. Comput., 32 (2010), pp. 111-128.

[38] P. Kloucek And F. S. Rys, Stability of the fractional step $\theta$-scheme for the nonstationary Navier-Stokes equations, SIAM J. Numer. Anal., 31 (1994), pp. 1312-1335.

[39] J. A. Lee, J. P. Rothstein, And M. Pasquali, Computational study of viscoelastic effects on liquid transfer during gravure printing, J. Non-Newtonian Fluid Mech., 199 (2013), pp. $1-11$.

Copyright (c) by SIAM. Unauthorized reproduction of this article is prohibited. 
[40] B. LindBerg, On smoothing and extrapolation for the trapezoidal rule, BIT, 11 (1971), pp. $29-52$.

[41] M. Luskin, R. Rannacher, And W. Wendland, On the smoothing property of the CrankNicolson scheme, Appl. Anal., 14 (1982), pp. 117-135.

[42] O. Østerby, Five ways of reducing the Crank-Nicolson oscillations, BIT, 43 (2003), pp. 811-822.

[43] M. Pasquali and L. E. Scriven, Free surface flows of polymer solutions with models based on the conformation tensor, J. Non-Newtonian Fluid Mech., 108 (2002), pp. 363-409.

[44] M. Pasquali and L. E. Scriven, Theoretical modeling of microstructured liquids: A simple thermodynamic approach, J. Non-Newtonian Fluid Mech., 120 (2004), pp. 101-136.

[45] S. V. Patankar and B. R. Baliga, A new finite-difference scheme for parabolic differential equations, Numer. Heat Transfer, 1 (1978), pp. 27-37.

[46] C. E. Pearson, Impulsive end condition for diffusion equation, Math. Comp., 19 (1965), pp. $570-576$.

[47] O. J. Romero and M. S. Carvalho, Response of slot coating flows to periodic disturbances, Chem. Eng. Sci., 63 (2008), pp. 2161-2173.

[48] O. J. Romero, L. E. Scriven, and M. S. Carvalho, Slot coating of mildly viscoelastic liquids, J. Non-Newtonian Fluid Mech., 138 (2006), pp. 63-75.

[49] L. F. Shampine and M. K. Gordon, Computer Solution of Ordinary Differential Equations: The Initial Value Problem, W. H. Freeman, New York, 1975.

[50] D. J. Silvester, A. Bespalov, and C. E. Powell, A framework for the development of implicit solvers for incompressible flow problems, Discrete Contin. Dyn. Syst. Ser. S, 32 (2012), pp. 1195-1221.

[51] J. C. Simo And F. Armero, Unconditional stability and long-term behavior of transient algorithms for the incompressible Navier-Stokes and Euler equations, Comput. Methods Appl. Mech. Engrg., 111 (1994), pp. 111-154.

[52] C. A. Smethurst, D. J. Silvester, And M. D. Minajlović, Unstructured finite element method for the solution of the Boussinesq problem in three dimensions, Internat. J. Numer. Methods Fluids, 73 (2013), pp. 791-812.

[53] J. Sorvari AND J. HämäLÄInen, Time integration in linear viscoelasticity - a comparative study, Mech. Time-Depend Mater., 14 (2010), pp. 307-328.

[54] D. G. Thomas, U. A. Al-Mubaiyedh, R. Sureshrumar, and B. Khomami, Time-dependent simulations of non-axisymmetric patterns in Taylor-Couette flow of dilute polymer solutions, J. Non-Newtonian Fluid Mech., 138 (2006), pp. 111-133.

[55] S. TureK, A comparative study of time-stepping techniques for the incompressible Navier-Stokes equations: From fully implicit non-linear schemes to semi-implicit projection methods, Internat. J. Numer. Methods Fluids, 22 (1996), pp. 987-1011.

[56] S. Turek, L. Rivkind, J. Hron, And R. Glowinski, Numerical study of a modified time-stepping $\theta$-scheme for incompressible flow simulations, J. Sci. Comput., 28 (2006), pp. $533-547$.

[57] E. D. Wilkes, S. D. Phillips, and O. A. Basaran, Computational and experimental analysis of dynamics of drop formation, Phys. Fluids, 11 (1999), p. 3577.

[58] W. L. Wood AND R. W. Lewis, A comparison of time marching schemes for the transient heat conduction equation, Internat. J. Numer. Methods Engrg., 9 (1975), pp. 679-689.

Copyright $@$ by SIAM. Unauthorized reproduction of this article is prohibited. 